J. Korean Math. Soc. 49 (2012), No. 1, pp. 187-200

http://dx.doi.org/10.4134/JKMS.2012.49.1.187

\title{
STRONG CONVERGENCE OF AN EXTENDED EXTRAGRADIENT METHOD FOR EQUILIBRIUM PROBLEMS AND FIXED POINT PROBLEMS
}

\author{
Jong Kyu Kim, Pham Ngoc Anh, and Young Man Nam
}

\begin{abstract}
In this paper, we introduced a new extended extragradient iteration algorithm for finding a common element of the set of fixed points of a nonexpansive mapping and the set of solutions of equilibrium problems for a monotone and Lipschitz-type continuous mapping. And we show that the iterative sequences generated by this algorithm converge strongly to the common element in a real Hilbert space.
\end{abstract}

\section{Introduction}

Let $C$ be a nonempty closed convex subset of a real Hilbert space $H$ and $f$ be a bifunction from $C \times C$ to $\mathbb{R}$. We consider the equilibrium problem: Find $x^{*} \in C$ such that

$E P(f, C) \quad f\left(x^{*}, y\right) \geq 0 \quad \forall y \in C$.

The set of solutions of $\operatorname{EP}(f, C)$ is denoted by $\operatorname{Sol}(f, C)$. These problems appear frequently in many practical problems arising, for instance, physics, engineering, game theory, transportation, economics and network, and become an attractive field for many researchers both theory and applications (see [1, $2,3,4,5,18,21])$.

If $f(x, y)=\langle F(x), y-x\rangle$ for every $x, y \in C$, where $F$ is a mapping from $C$ to $H$, then the problem $E P(f, C)$ becomes the following variational inequality: Find $x^{*} \in C$ such that

$V I(F, C) \quad\left\langle F\left(x^{*}\right), y-x^{*}\right\rangle \geq 0 \quad \forall y \in C$.

We denote $\operatorname{Sol}(F, C)$ which is the set of solutions of $V I(F, C)$.

Received October 3, 2010.

2010 Mathematics Subject Classification. 65K10, 90C25.

Key words and phrases. equilibrium problems, monotone mapping, Lipschitz-type continuous, strong convergence, extragradient algorithm, nonexpansive mapping.

This work was supported by National Research Foundation of Korea Grant funded by the Korean Government (2009-0076898), was completed while the second author was staying at the Kyungnam University. 
For solving $V I(F, C)$ in the Euclidean space $\mathbb{R}^{n}$ under the assumption that a subset $C \subseteq \mathbb{R}^{n}$ is nonempty closed convex, $F$ is monotone, $L$-Lipschitz continuous and $\operatorname{Sol}(F, C) \neq \emptyset$, Korpelevich in [9] introduced the following extragradient method:

$$
\left\{\begin{array}{l}
x^{0} \in C, \\
y^{k}=\operatorname{Pr}_{C}\left(x^{k}-\lambda F\left(x^{k}\right)\right), \\
x^{k+1}=\operatorname{Pr}_{C}\left(x^{k}-\lambda F\left(y^{k}\right)\right),
\end{array}\right.
$$

for all $k \geq 0$, where $\lambda \in\left(0, \frac{1}{L}\right)$ and $\operatorname{Pr}_{C}$ is denoted the projection on $C$. The author showed that the sequences $\left\{x^{k}\right\}$ and $\left\{y^{k}\right\}$ converge to the same point $\bar{x} \in \operatorname{Sol}(F, C)$.

Takahashi and Toyoda in [17] introduced an extragradient method for finding a common element of $\operatorname{Sol}(F, C)$ and the set of fixed points of a nonexpansive mapping $T$ (shortly $F i x(T)$ ) under the assumption that a subset $C \subseteq H$ is closed convex and $F$ is $\alpha$-inverse strongly monotone:

$$
\left\{\begin{array}{l}
x^{0} \in C, \\
x^{k+1}=\alpha_{k} x^{k}+\left(1-\alpha_{k}\right) \operatorname{TPr}_{C}\left(x^{k}-\lambda_{k} F\left(x^{k}\right)\right),
\end{array}\right.
$$

for all $k \geq 0$, where $\left\{\alpha_{k}\right\}$ is a sequence in $(0,1)$ and $\left\{\lambda_{k}\right\}$ is a sequence in $(0,2 \alpha)$. They proved that if $\operatorname{Fix}(T) \cap \operatorname{Sol}(F, C) \neq \emptyset$, then the sequence $\left\{x^{k}\right\}$ converges weakly to some $\bar{x} \in \operatorname{Sol}(F, C) \cap F i x(T)$.

For obtaining a common element of $\operatorname{Sol}(f, C)$ and the set of fixed points of a nonexpansive mapping $T$, Takahashi and Takahashi in [16] introduced an iterative scheme by the viscosity approximation method. Sequences $\left\{x^{k}\right\}$ and $\left\{y^{k}\right\}$ are defined by:

$$
\left\{\begin{array}{l}
x^{0} \in H \\
f\left(y^{k}, y\right)+\frac{1}{r_{k}}\left\langle y-y^{k}, y^{k}-x^{k}\right\rangle \geq 0 \quad \forall y \in C, \\
x^{k+1}=\alpha_{k} g\left(x^{k}\right)+\left(1-\alpha_{k}\right) T\left(y^{k}\right) \quad \forall k \geq 0 .
\end{array}\right.
$$

The authors showed that under certain conditions over $\left\{\alpha_{k}\right\}$ and $\left\{r_{k}\right\}$, sequences $\left\{x^{k}\right\}$ and $\left\{y^{k}\right\}$ converge strongly to $z=\operatorname{Pr}_{F i x(T) \cap \operatorname{Sol}(f, C)}(g(z))$.

Recently, iterative algorithms for finding a common element of the set of solutions of equilibrium problems and the set of fixed points of a nonexpansive mapping in a real Hilbert space have further developed by some authors (see $[6,7,8,10,12,14,15,16,18,21,22])$. At each iteration $k$ in all of these algorithms, it requires solving approximation auxiliary equilibrium problems.

In this paper, we introduce a new iterative algorithm for finding a common element of the set of fixed points of a nonexpansive mapping and the set of solutions of equilibrium problems for a monotone, Lipschitz-type continuous bifunction. At each iteration $k$, we only solve strongly convex problems on $C$. The iterative process is based on so-called extragradient method. We obtain a strong convergence theorem for three sequences generated by this process. 


\section{Preliminaries}

Let $H$ be a real Hilbert space with inner product $\langle\cdot, \cdot\rangle$ and norm $\|\cdot\|$, respectively. We list some well known definitions.

Definition 2.1. Let $C$ be a nonempty closed convex subset of $H$.

(I) The bifunction $f: C \times C \rightarrow \mathbb{R}$ is said to be

(i) $\gamma$-strongly monotone on $C$ if for each $x, y \in C$,

$$
f(x, y)+f(y, x) \leq-\gamma\|x-y\|^{2} ;
$$

(ii) monotone on $C$ if for each $x, y \in C$,

$$
f(x, y)+f(y, x) \leq 0
$$

(iii) pseudomonotone on $C$ if for each $x, y \in C$,

$$
f(x, y) \geq 0 \Rightarrow f(y, x) \leq 0
$$

(iv) Lipschitz-type continuous on $C$ with constants $c_{1}>0$ and $c_{2}>0$, if for each $x, y \in C$,

$$
f(x, y)+f(y, z) \geq f(x, z)-c_{1}\|x-y\|^{2}-c_{2}\|y-z\|^{2} .
$$

(II) The mapping $F: C \rightarrow H$ is said to be

(i) monotone on $C$ if for each $x, y \in C$,

$$
\langle F(x)-F(y), x-y\rangle \geq 0 ;
$$

(ii) pseudomonotone on $C$ if for each $x, y \in C$,

$$
\langle F(y), x-y\rangle \geq 0 \Rightarrow\langle F(x), x-y\rangle \geq 0 ;
$$

(iii) $L$-Lipschitz continuous on $C$ if for each $x, y \in C$,

$$
\|F(x)-F(y)\| \leq L\|x-y\| .
$$

If $L=1$, then $F$ is nonexpansive on $C$.

Now, we define the projection on $C$, denoted by $\operatorname{Pr}_{C}(\cdot)$, i.e.,

$$
\operatorname{Pr}_{C}(x)=\operatorname{argmin}\{\|y-x\|: y \in C\} \quad \forall x \in H .
$$

A space $X$ is said to have Opial's condition ([13]) if for any sequence $\left\{x_{n}\right\}$ with $x_{n} \rightarrow \bar{x}$, the inequality

$$
\liminf _{n \rightarrow \infty}\left\|x_{n}-\bar{x}\right\|<\liminf _{n \rightarrow \infty}\left\|x_{n}-y\right\|
$$

holds for every $y \in H$ with $y \neq \bar{x}$,

Note that if $F$ is $L$-Lipschitz on $C$, then for each $x, y \in C, f(x, y)=$ $\langle F(x), y-x)$ is Lipschitz-type continuous with constants $c_{1}=c_{2}=\frac{L}{2}$ on $C$. Indeed,

$$
\begin{aligned}
f(x, y)+f(y, z)-f(x, z) & =\langle F(x), y-x\rangle+\langle F(y), z-y\rangle+\langle F(x), z-x\rangle \\
& =-\langle F(y)-F(x), y-z\rangle \\
& \geq-\|F(x)-F(y)\|\|y-z\|
\end{aligned}
$$




$$
\begin{aligned}
& \geq-L\|x-y\|\|y-z\| \\
& \geq-\frac{L}{2}\|x-y\|^{2}-\frac{L}{2}\|y-z\|^{2} \\
& =-c_{1}\|x-y\|^{2}-c_{2}\|y-z\|^{2} .
\end{aligned}
$$

Thus $f$ is Lipschitz-type continuous on $C$.

In this paper, for finding a point of the set $\operatorname{Sol}(f, C) \cap F i x(T)$, we assume that the bifunction $f$ satisfies the following conditions:

(i) $f$ is monotone on $C$;

(ii) $f$ is Lipschitz-type continuous on $C$;

(iii) for each $x \in C, y \mapsto f(x, y)$ is convex and subdifferentiable on $C$;

(iv) $f$ is upper semicontinuous on $C$;

(v) $\operatorname{Sol}(f, C) \cap \operatorname{Fix}(T) \neq \emptyset$.

Now we are in a position to describe the extended extragradient algorithm for finding a common element of $\operatorname{Sol}(f, C) \cap F i x(T)$.

Algorithm 2.2. Choose $u \in H$, positive sequences $\left\{\lambda_{n}\right\},\left\{\alpha_{n}\right\},\left\{\beta_{n}\right\}$ and $\left\{\gamma_{n}\right\}$ satisfy the conditions:

$$
\left\{\begin{array}{l}
\left\{\lambda_{n}\right\} \subset\left(0, \min \left\{\frac{1}{2 c_{1}}, \frac{1}{2 c_{2}}\right\}\right), \lim _{n \rightarrow \infty} \lambda_{n}=\lambda \in\left(0, \frac{2 \delta-1}{4 c_{2}}\right], \\
\alpha_{n}+\beta_{n}+\gamma_{n}=1, \lim _{n \rightarrow \infty} \alpha_{n}=0, \sum_{n=0}^{\infty} \alpha_{n}=\infty, \lim _{n \rightarrow \infty} \beta_{n}=\beta \in(0,1) .
\end{array}\right.
$$

Step 1. Solve the strongly convex problems:

$$
\left\{\begin{array}{l}
y^{n}:=\operatorname{argmin}\left\{\frac{1}{2}\left\|y-x^{n}\right\|^{2}+\lambda_{n} f\left(x^{n}, y\right): y \in C\right\}, \\
t^{n}:=\operatorname{argmin}\left\{\frac{1}{2}\left\|t-x^{n}\right\|^{2}+\lambda_{n} f\left(y^{n}, t\right): t \in C\right\} .
\end{array}\right.
$$

Step 2. Set $x^{n+1}:=\alpha_{n} u+\beta_{n} x^{n}+\gamma_{n} T\left(t^{n}\right)$.

Increase $k$ by 1 and go to Step 1 .

In order to prove the main result in Section 3, we shall use the following lemmas in the sequel.

Lemma 2.3 (see [5]). Let $C$ be a nonempty closed convex subset of a real Hilbert space $H$ and $g: C \rightarrow \mathbb{R}$ be convex and subdifferentiable on $C$. Then $x^{*}$ is a solution to the following convex problem

$$
\min \{g(x): x \in C\}
$$

if and only if

$$
0 \in \partial g\left(x^{*}\right)+N_{C}\left(x^{*}\right)
$$

where $\partial g(\cdot)$ denotes the subdifferential of $g$ and $N_{C}\left(x^{*}\right)$ is the (outward) normal cone of $C$ at $x^{*} \in C$.

Lemma 2.4 (see [11]). Assume that $T$ is a nonexpansive self-mapping of a nonempty closed convex subset $C$ of a real Hilbert space $H$. If Fix $(T) \neq \emptyset$, then $I-T$ is demiclosed; that is, whenever $\left\{x^{n}\right\}$ is a sequence in $C$ weakly 
converging to some $\bar{x} \in C$ and the sequence $\left\{(I-T)\left(x^{n}\right)\right\}$ strongly converges to some $\bar{y}$, it follows that $(I-T)(\bar{x})=\bar{y}$. Here $I$ is the identity operator of $H$.

Lemma 2.5 (see [20]). Let $\left\{x^{n}\right\}$ and $\left\{y^{n}\right\}$ be bounded sequences in a Banach space $X$ and let $\left\{\beta_{n}\right\}$ be a sequence in $[0,1]$ with

$$
0<\liminf _{n \rightarrow \infty} \beta_{n} \leq \limsup _{n \rightarrow \infty} \beta_{n}<1 .
$$

Suppose

$x^{n+1}=\left(1-\beta_{n}\right) y^{n}+\beta_{n} x^{n} \forall n \geq 0$ and $\limsup _{n \rightarrow \infty}\left(\left\|y^{n+1}-y^{n}\right\|-\left\|x^{n+1}-x^{n}\right\|\right) \leq 0$.

Then, $\lim _{n \rightarrow \infty}\left\|y^{n}-x^{n}\right\|=0$.

Lemma 2.6 (see [19]). Let $\left\{a_{n}\right\}$ be a sequence of nonnegative real numbers such that

$$
a_{n+1} \leq\left(1-\gamma_{n}\right) a_{n}+\delta_{n}
$$

where $\left\{\gamma_{n}\right\}$ is a sequence in $(0,1)$ and $\left\{\delta_{n}\right\}$ is a sequence such that

$$
\left\{\begin{array}{l}
\sum_{n=1}^{\infty} \gamma_{n}=\infty \\
\limsup _{n \rightarrow \infty} \frac{\delta_{n}}{\gamma_{n}} \leq 0 \text { or } \quad \sum_{n=1}^{\infty}\left|\delta_{n}\right|<\infty
\end{array}\right.
$$

Then $\lim _{n \rightarrow \infty} a_{n}=0$.

\section{Main results}

In this section, we prove that the strong convergence of the sequences $\left\{x^{n}\right\}$, $\left\{y^{n}\right\}$ and $\left\{t^{n}\right\}$ defined by Algorithm 2.2 based on the extragradient method which solves the problem of finding a common element of two sets $\operatorname{Sol}(f, C)$ and $\operatorname{Fix}(T)$ for a monotone, Lipschitz-type continuous bifunction $f$ in a real Hilbert space $H$.

Lemma 3.1. Let $f(x, \cdot)$ be convex and subdifferentiable on $C$ for all $x \in C$, and $f$ be pseudomonotone on $C$. Then for $x^{*} \in \operatorname{Sol}(f, C)$, we have

$\left\|t^{n}-x^{*}\right\|^{2} \leq\left\|x^{n}-x^{*}\right\|^{2}-\left(1-2 \lambda_{n} c_{2}\right)\left\|t^{n}-y^{n}\right\|^{2}-\left(1-2 \lambda_{n} c_{1}\right)\left\|x^{n}-y^{n}\right\|^{2} \forall n \geq 0$.

Proof. Since $f(x, \cdot)$ is convex on $C$ for each $x \in C$ and Lemma 2.3, we obtain

$$
t^{n}=\operatorname{argmin}\left\{\frac{1}{2}\left\|t-x^{n}\right\|^{2}+\lambda_{n} f\left(y^{n}, t\right): t \in C\right\}
$$

if and only if

$$
0 \in \partial_{2}\left\{\lambda_{n} f\left(y^{n}, y\right)+\frac{1}{2}\left\|y-x^{n}\right\|^{2}\right\}\left(t^{n}\right)+N_{C}\left(t^{n}\right) .
$$

Since $f\left(y^{n}, \cdot\right)$ is subdifferentiable on $C$, by the well known Moreau-Rockafellar Theorem (see [5]), there exists $w \in \partial_{2} f\left(y^{n}, t^{n}\right)$ such that

$$
f\left(y^{n}, t\right)-f\left(y^{n}, t^{n}\right) \geq\left\langle w, t-t^{n}\right\rangle \quad \forall t \in C .
$$


For $t=x^{*} \in C$, this inequality becomes

$$
f\left(y^{n}, x^{*}\right)-f\left(y^{n}, t^{n}\right) \geq\left\langle w, x^{*}-t^{n}\right\rangle .
$$

From (3.1), it follows that

$$
0=\lambda_{n} w+t^{n}-x^{n}+\bar{w}
$$

where $w \in \partial_{2} f\left(y^{n}, t^{n}\right)$ and $\bar{w} \in N_{C}\left(t^{n}\right)$. From the last inequality and the definition of the normal cone $N_{C}$, we have

$$
\left\langle t^{n}-x^{n}, t-t^{n}\right\rangle \geq \lambda_{n}\left\langle w, t^{n}-t\right\rangle \quad \forall t \in C .
$$

Using $t=x^{*} \in C$, we obtain

$$
\left\langle t^{n}-x^{n}, x^{*}-t^{n}\right\rangle \geq \lambda_{n}\left\langle w, t^{n}-x^{*}\right\rangle .
$$

It follows from (3.3) and (3.5) that

$$
\left\langle t^{n}-x^{n}, x^{*}-t^{n}\right\rangle \geq \lambda_{n}\left(f\left(y^{n}, t^{n}\right)-f\left(y^{n}, x^{*}\right)\right) .
$$

Since $x^{*} \in \operatorname{Sol}(f, C), f\left(x^{*}, y\right) \geq 0$ for all $y \in C$, and $f$ is pseudomonotone on $C$, we have $f\left(y^{n}, x^{*}\right) \leq 0$. Then, (3.6) implies that

$$
\left\langle t^{n}-x^{n}, x^{*}-t^{n}\right\rangle \geq \lambda_{n} f\left(y^{n}, t^{n}\right) .
$$

Now applying Lipschitzian of $f$ with $x=x^{n}, y=y^{n}$ and $z=t^{n}$, we get

$$
f\left(y^{n}, t^{n}\right) \geq f\left(x^{n}, t^{n}\right)-f\left(x^{n}, y^{n}\right)-c_{1}\left\|y^{n}-x^{n}\right\|^{2}-c_{2}\left\|t^{n}-y^{n}\right\|^{2} .
$$

Combinating (3.7) and (3.8), we have

(3.9) $\left\langle t^{n}-x^{n}, x^{*}-t^{n}\right\rangle \geq \lambda_{n}\left(f\left(x^{n}, t^{n}\right)-f\left(x^{n}, y^{n}\right)-c_{1}\left\|y^{n}-x^{n}\right\|^{2}-c_{2}\left\|t^{n}-y^{n}\right\|^{2}\right)$.

Similarly, since $y^{n}$ is the unique solution to the strongly convex problem

$$
\min \left\{\frac{1}{2}\left\|y-x^{n}\right\|^{2}+\lambda_{n} f\left(x^{n}, y\right): y \in C\right\},
$$

we have

$$
\lambda_{n}\left(f\left(x^{n}, y\right)-f\left(x^{n}, y^{n}\right)\right) \geq\left\langle y^{n}-x^{n}, y^{n}-y\right\rangle \quad \forall y \in C .
$$

Substituting $y=t^{n} \in C$, we obtain

$$
\lambda_{n}\left(f\left(x^{n}, t^{n}\right)-f\left(x^{n}, y^{n}\right)\right) \geq\left\langle y^{n}-x^{n}, y^{n}-t^{n}\right\rangle .
$$

From (3.9), (3.11) and

$$
2\left\langle t^{n}-x^{n}, x^{*}-t^{n}\right\rangle=\left\|x^{n}-x^{*}\right\|^{2}-\left\|t^{n}-x^{n}\right\|^{2}-\left\|t^{n}-x^{*}\right\|^{2},
$$

it implies that

$$
\begin{aligned}
& \left\|x^{n}-x^{*}\right\|^{2}-\left\|t^{n}-x^{n}\right\|^{2}-\left\|t^{n}-x^{*}\right\|^{2} \\
\geq & 2\left\langle y^{n}-x^{n}, y^{n}-t^{n}\right\rangle-2 \lambda_{n} c_{1}\left\|x^{n}-y^{n}\right\|^{2}-2 \lambda_{n} c_{2}\left\|t^{n}-y^{n}\right\|^{2} .
\end{aligned}
$$

Hence, we have

$$
\begin{aligned}
& \left\|t^{n}-x^{*}\right\|^{2} \\
\leq & \left\|x^{n}-x^{*}\right\|^{2}-\left\|t^{n}-x^{n}\right\|^{2}-2\left\langle y^{n}-x^{n}, y^{n}-t^{n}\right\rangle+2 \lambda_{n} c_{1}\left\|x^{n}-y^{n}\right\|^{2}
\end{aligned}
$$




$$
\begin{aligned}
& +2 \lambda_{n} c_{2}\left\|t^{n}-y^{n}\right\|^{2} \\
= & \left\|x^{n}-x^{*}\right\|^{2}-\left\|\left(t^{n}-y^{n}\right)+\left(y^{n}-x^{n}\right)\right\|^{2}-2\left\langle y^{n}-x^{n}, y^{n}-t^{n}\right\rangle \\
& +2 \lambda_{n} c_{1}\left\|x^{n}-y^{n}\right\|^{2}+2 \lambda_{n} c_{2}\left\|t^{n}-y^{n}\right\|^{2} \\
\leq & \left\|x^{n}-x^{*}\right\|^{2}-\left\|t^{n}-y^{n}\right\|^{2}-\left\|x^{n}-y^{n}\right\|^{2}+2 \lambda_{n} c_{1}\left\|x^{n}-y^{n}\right\|^{2}+2 \lambda_{n} c_{2}\left\|t^{n}-y^{n}\right\|^{2} \\
= & \left\|x^{n}-x^{*}\right\|^{2}-\left(1-2 \lambda_{n} c_{1}\right)\left\|x^{n}-y^{n}\right\|^{2}-\left(1-2 \lambda_{n} c_{2}\right)\left\|y^{n}-t^{n}\right\|^{2} .
\end{aligned}
$$

This completes the proof.

Lemma 3.2. Suppose that assumptions (i)-(v) hold and $T$ is nonexpansive on $C$, for each $x \in C, f(x, \cdot)$ is strongly convex with constant $\delta>0$ on $C$. Then the sequences $\left\{x^{n}\right\},\left\{y^{n}\right\}$ and $\left\{t^{n}\right\}$ generated by Algorithm 2.2 satisfy

$$
\left\|x^{n+1}-x^{*}\right\|^{2} \leq \alpha_{n}\left\|u-x^{*}\right\|^{2}+\left\|x^{n}-x^{*}\right\|^{2}-\left(1-2 \lambda_{n} c_{1}\right) \gamma_{n}\left\|x^{n}-y^{n}\right\|^{2}
$$

$$
-\left(1-2 \lambda_{n} c_{2}\right) \gamma_{n}\left\|t^{n}-y^{n}\right\|^{2} \text {. }
$$

Consequently,

provided $\lim _{n \rightarrow \infty}\left\|x^{n}-y^{n}\right\|=0$.

$$
\lim _{n \rightarrow \infty}\left\|x^{n+1}-x^{n}\right\|=\lim _{n \rightarrow \infty}\left\|t^{n}-y^{n}\right\|=0,
$$

Proof. For each $n$, it follows from (3.4) that

$$
\left\langle t^{n}-x^{n}, t-t^{n}\right\rangle \geq \lambda_{n}\left\langle w, t^{n}-t\right\rangle \quad \forall w \in \partial_{2} f\left(y^{n}, t^{n}\right), t \in C .
$$

With $t=y^{n} \in C$, we have

$$
\left\langle t^{n}-x^{n}, y^{n}-t^{n}\right\rangle \geq \lambda_{n}\left\langle w, t^{n}-y^{n}\right\rangle \quad \forall w \in \partial_{2} f\left(y^{n}, t^{n}\right) .
$$

Combining $f(x, x)=0$ for all $x \in C$, the last inequality and the definition of $w$,

$$
f\left(y^{n}, t\right)-f\left(y^{n}, t^{n}\right) \geq\left\langle w, t-t^{n}\right\rangle \quad \forall t \in C,
$$

we have

$$
\begin{aligned}
\left\langle t^{n}-x^{n}, y^{n}-t^{n}\right\rangle & \geq-\lambda_{n}\left\langle w, y^{n}-t^{n}\right\rangle \\
& \geq \lambda_{n}\left(f\left(y^{n}, t^{n}\right)-f\left(y^{n}, y^{n}\right)\right) \\
& =\lambda_{n} f\left(y^{n}, t^{n}\right) .
\end{aligned}
$$

Substituting $y=t^{n} \in C$ into (3.10), we get

$$
\left\langle y^{n}-x^{n}, t^{n}-y^{n}\right\rangle \geq \lambda_{n}\left(f\left(x^{n}, y^{n}\right)-f\left(x^{n}, t^{n}\right)\right) .
$$

Adding two inequalities (3.13) and (3.14), we obtain

$$
\left\langle t^{n}-y^{n}, y^{n}-x^{n}-t^{n}+x^{n}\right\rangle \geq \lambda_{n}\left(f\left(x^{n}, y^{n}\right)+f\left(y^{n}, t^{n}\right)-f\left(x^{n}, t^{n}\right)\right) .
$$

Then, since $f$ is Lipschitz-type continuous on $C$, we have

$$
-\left\|t^{n}-y^{n}\right\|^{2} \geq \lambda_{n}\left(-c_{1}\left\|x^{n}-y^{n}\right\|^{2}-c_{2}\left\|y^{n}-t^{n}\right\|^{2}\right),
$$

which follows that

$$
\left(1-\lambda_{n} c_{2}\right)\left\|t^{n}-y^{n}\right\|^{2} \leq \lambda_{n} c_{1}\left\|x^{n}-y^{n}\right\|^{2} .
$$


So, we get

$$
\left\|t^{n}-y^{n}\right\|^{2} \leq \frac{\lambda_{n} c_{1}}{1-\lambda_{n} c_{2}}\left\|x^{n}-y^{n}\right\|^{2} .
$$

For each $x^{*} \in \operatorname{Sol}(f, C) \cap \operatorname{Fix}(T)$, from Lemma 3.1, it implies that

$$
\begin{aligned}
\left\|x^{n+1}-x^{*}\right\|^{2} & =\left\|\alpha_{n} u+\beta_{n} x^{n}+\gamma_{n} T\left(t^{n}\right)-x^{*}\right\|^{2} \\
& =\left\|\alpha_{n}\left(u-x^{*}\right)+\beta_{n}\left(x^{n}-x^{*}\right)+\gamma_{n}\left(T\left(t^{n}\right)-x^{*}\right)\right\|^{2} \\
& \leq \alpha_{n}\left\|u-x^{*}\right\|^{2}+\beta_{n}\left\|x^{n}-x^{*}\right\|^{2}+\gamma_{n}\left\|T\left(t^{n}\right)-T\left(x^{*}\right)\right\|^{2} \\
& \leq \alpha_{n}\left\|u-x^{*}\right\|^{2}+\beta_{n}\left\|x^{n}-x^{*}\right\|^{2}+\gamma_{n}\left\|t^{n}-x^{*}\right\|^{2} \\
& \leq \alpha_{n}\left\|u-x^{*}\right\|^{2}+\beta_{n}\left\|x^{n}-x^{*}\right\|^{2}+\gamma_{n}\left\|x^{n}-x^{*}\right\|^{2} \\
& =\alpha_{n}\left\|u-x^{*}\right\|^{2}+\left(1-\alpha_{n}\right)\left\|x^{n}-x^{*}\right\|^{2} \\
& \leq \max \left\{\left\|u-x^{*}\right\|^{2},\left\|u-x^{*}\right\|^{2}\right\} .
\end{aligned}
$$

Therefore $\left\{x^{n}\right\}$ is bounded and it follows from Lemma 3.1 that $\left\{x^{n}\right\},\left\{t^{n}\right\}$, $\left\{y^{n}\right\}$ are bounded. Since $f(x, \cdot)$ is $\delta$-strongly convex on $C$ for all $x \in C$, we have

$$
f\left(y^{n}, t^{n+1}\right)-f\left(y^{n}, t^{n}\right) \geq\left\langle w, t^{n+1}-t^{n}\right\rangle+\frac{\delta}{2}\left\|t^{n+1}-t^{n}\right\|^{2},
$$

where $w \in \partial_{2} f\left(y^{n}, t^{n}\right)$. Substituting $t=t^{n+1}$ into (3.4), then we have

$$
\left\langle t^{n}-x^{n}, t^{n+1}-t^{n}\right\rangle \geq \lambda_{n}\left\langle w, t^{n}-t^{n+1}\right\rangle
$$

$$
\geq \lambda_{n}\left(f\left(y^{n}, t^{n}\right)-f\left(y^{n}, t^{n+1}\right)\right)+\frac{\lambda_{n} \delta}{2}\left\|t^{n+1}-t^{n}\right\|^{2} .
$$

Similarly, we also have

$$
\left\langle t^{n+1}-x^{n+1}, t^{n}-t^{n+1}\right\rangle
$$

Adding (3.18) and (3.19), we get

$$
\begin{aligned}
& \left\langle t^{n+1}-t^{n}, t^{n}-x^{n}-t^{n+1}+x^{n+1}\right\rangle \\
\geq & \lambda_{n}\left(f\left(y^{n}, t^{n}\right)-f\left(y^{n}, t^{n+1}\right)\right)+\frac{\lambda_{n} \delta}{2}\left\|t^{n+1}-t^{n}\right\|^{2} \\
& +\lambda_{n+1}\left(f\left(y^{n+1}, t^{n+1}\right)-f\left(y^{n+1}, t^{n}\right)\right)+\frac{\lambda_{n+1} \delta}{2}\left\|t^{n+1}-t^{n}\right\|^{2} .
\end{aligned}
$$

From monotonicity and Lipschitz-type continuity of $f$ and $\langle x, y\rangle \leq \frac{1}{2}\left(\|x\|^{2}+\right.$ $\left.\left\|y^{2}\right\|\right)$ for all $x, y \in H$, it implies that

$$
\begin{aligned}
& \frac{1}{2}\left(\left\|t^{n+1}-t^{n}\right\|^{2}-\left\|x^{n+1}-x^{n}\right\|^{2}\right) \\
\leq & \left\|t^{n+1}-t^{n}\right\|^{2}-\left\langle t^{n+1}-t^{n}, x^{n+1}-x^{n}\right\rangle \\
= & -\left\langle t^{n+1}-t^{n}, t^{n}-x^{n}-t^{n+1}+x^{n+1}\right\rangle
\end{aligned}
$$




$$
\begin{aligned}
\leq & \lambda_{n}\left(f\left(y^{n}, t^{n+1}\right)-f\left(y^{n}, t^{n}\right)\right)-\frac{\lambda_{n} \delta}{2}\left\|t^{n+1}-t^{n}\right\|^{2} \\
& +\lambda_{n+1}\left(f\left(y^{n+1}, t^{n}\right)-f\left(y^{n+1}, t^{n+1}\right)\right)-\frac{\lambda_{n+1} \delta}{2}\left\|t^{n+1}-t^{n}\right\|^{2} \\
\leq & \lambda_{n}\left(f\left(t^{n}, t^{n+1}\right)+c_{1}\left\|y^{n}-t^{n}\right\|^{2}+\left(c_{2}-\frac{\delta}{2}\right)\left\|t^{n+1}-t^{n}\right\|^{2}\right) \\
& +\lambda_{n+1}\left(f\left(t^{n+1}, t^{n}\right)+c_{1}\left\|y^{n+1}-t^{n+1}\right\|^{2}+\left(c_{2}-\frac{\delta}{2}\right)\left\|t^{n+1}-t^{n}\right\|^{2}\right) \\
\leq & \left.\left(\lambda_{n}-\lambda_{n+1}\right) f\left(t^{n}, t^{n+1}\right)+\left(\lambda_{n}+\lambda_{n+1}\right) c_{2}-\delta\right)\left\|t^{n+1}-t^{n}\right\|^{2}+c_{1} \lambda_{n}\left\|y^{n}-t^{n}\right\|^{2} \\
& +c_{1} \lambda_{n+1}\left\|y^{n+1}-t^{n+1}\right\|^{2} .
\end{aligned}
$$

Then we have

$m_{n}\left\|t^{n+1}-t^{n}\right\|^{2} \leq\left\|x^{n+1}-x^{n}\right\|^{2}+2\left(\lambda_{n}-\lambda_{n+1}\right) f\left(t^{n}, t^{n+1}\right)+2 c_{1} \lambda_{n}\left\|y^{n}-t^{n}\right\|^{2}$

$$
+2 c_{1} \lambda_{n+1}\left\|y^{n+1}-t^{n+1}\right\|^{2},
$$

where $m_{n}=1+2 \delta-2\left(\lambda_{n}+\lambda_{n+1}\right) c_{2}$. It follows from $\lambda \leq \frac{2 \delta-1}{4 c_{2}}$ that there exists $n_{0}$ such that $m_{n}>0$ for all $n \geq n_{0}$ and we have

$$
\begin{aligned}
& \frac{\gamma_{n+1}^{2}}{\left(1-\beta_{n+1}\right)^{2}}\left\|t^{n+1}-t^{n}\right\|^{2}-\frac{1}{2}\left\|x^{n+1}-x^{n}\right\|^{2} \\
\leq & M_{n}\left\|x^{n+1}-x^{n}\right\|^{2}+\frac{2 \gamma_{n+1}^{2}\left(\lambda_{n}-\lambda_{n+1}\right)}{m_{n}\left(1-\beta_{n+1}\right)^{2}} f\left(t^{n}, t^{n+1}\right) \\
& +\frac{2 c_{1} \lambda_{n} \gamma_{n+1}^{2}}{\left(1-\beta_{n+1}\right)^{2}}\left\|y^{n}-t^{n}\right\|^{2}+\frac{2 c_{1} \lambda_{n+1} \gamma_{n+1}^{2}}{\left(1-\beta_{n+1}\right)^{2}}\left\|y^{n+1}-t^{n+1}\right\|^{2},
\end{aligned}
$$

where

$$
M_{n}=\frac{\gamma_{n+1}^{2}}{m_{n}\left(1-\beta_{n+1}\right)^{2}}-\frac{1}{2} .
$$

From (iv), (3.21), Lemma 3.1, (3.17), (3.16), $\lim _{n \rightarrow \infty}\left\|x^{n}-y^{n}\right\|=0$ and

$$
\lim _{n \rightarrow \infty} M_{n}=\frac{1-2 \delta+4 \lambda c_{2}}{2\left(1+2 \delta-4 \lambda c_{2}\right)} \leq 0,
$$

we have

$$
\lim _{n \rightarrow \infty}\left(\frac{\gamma_{n+1}^{2}}{\left(1-\beta_{n+1}\right)^{2}}\left\|t^{n+1}-t^{n}\right\|^{2}-\frac{1}{2}\left\|x^{n+1}-x^{n}\right\|^{2}\right) \leq 0 .
$$

Set $x^{n+1}=\left(1-\beta_{n}\right) z^{n}+\beta_{n} x^{n}$. Then, we obtain

$$
\begin{aligned}
z^{n+1}-z^{n}= & \frac{\alpha_{n+1} u+\gamma_{n+1} T\left(t^{n+1}\right)}{1-\beta_{n+1}}-\frac{\alpha_{n} u+\gamma_{n} T\left(t^{n}\right)}{1-\beta_{n}} \\
= & \left(\frac{\alpha_{n+1}}{1-\beta_{n+1}}-\frac{\alpha_{n}}{1-\beta_{n}}\right) u+\frac{\gamma_{n+1}}{1-\beta_{n+1}}\left(T\left(t^{n+1}\right)-T\left(t^{n}\right)\right) \\
& +\left(\frac{\gamma_{n+1}}{1-\beta_{n+1}}-\frac{\gamma_{n}}{1-\beta_{n}}\right) T\left(t^{n}\right) .
\end{aligned}
$$


Hence, we have

$$
\begin{aligned}
& \frac{1}{2}\left(\left\|z^{n+1}-z^{n}\right\|^{2}-\left\|x^{n+1}-x^{n}\right\|^{2}\right) \\
\leq & \left|\frac{\alpha_{n+1}}{1-\beta_{n+1}}-\frac{\alpha_{n}}{1-\beta_{n}}\right|^{2}\|u\|^{2}+\left(\frac{\gamma_{n+1}}{1-\beta_{n+1}}\right)^{2}\left\|t^{n+1}-t^{n}\right\|^{2} \\
& +\left|\frac{\gamma_{n+1}}{1-\beta_{n+1}}-\frac{\gamma_{n}}{1-\beta_{n}}\right|^{2}\left\|T\left(t^{n}\right)\right\|^{2}-\frac{1}{2}\left\|x^{n+1}-x^{n}\right\|^{2},
\end{aligned}
$$

Combining this, (3.23), and boundedness of the sequences $\left\{x^{n}\right\},\left\{y^{n}\right\},\left\{t^{n}\right\}$ and $\left\{T\left(t^{n}\right)\right\}$, we obtain

$$
\limsup _{n \rightarrow \infty}\left(\left\|z^{n+1}-z^{n}\right\|-\left\|x^{n+1}-x^{n}\right\|\right) \leq 0 .
$$

Hence by Lemma 2.5, we obtain $\lim _{n \rightarrow \infty}\left\|z^{n}-x^{n}\right\|=0$. Consequently,

$$
\lim _{n \rightarrow \infty}\left\|x^{n+1}-x^{n}\right\|=\lim _{n \rightarrow \infty}\left(1-\beta_{n}\right)\left\|z^{n}-x^{n}\right\|=0 .
$$

It follows from (3.20) that $\lim _{n \rightarrow \infty}\left\|t^{n+1}-t^{n}\right\|=0$. By (3.17) and Lemma 3.1, we have

$$
\begin{aligned}
& \left\|x^{n+1}-x^{*}\right\|^{2} \\
\leq & \alpha_{n}\left\|u-x^{*}\right\|^{2}+\beta_{n}\left\|x^{n}-x^{*}\right\|^{2}+\gamma_{n}\left\|x^{n}-x^{*}\right\|^{2}-\left(1-2 \lambda_{n} c_{2}\right) \gamma_{n}\left\|t^{n}-y^{n}\right\|^{2} \\
& -\left(1-2 \lambda_{n} c_{1}\right) \gamma_{n}\left\|x^{n}-y^{n}\right\|^{2} \\
\leq & \alpha_{n}\left\|u-x^{*}\right\|^{2}+\left\|x^{n}-x^{*}\right\|^{2}-\left(1-2 \lambda_{n} c_{2}\right) \gamma_{n}\left\|t^{n}-y^{n}\right\|^{2} \\
& -\left(1-2 \lambda_{n} c_{1}\right) \gamma_{n}\left\|x^{n}-y^{n}\right\|^{2} .
\end{aligned}
$$

This implies (3.12) and

$$
\begin{aligned}
& \left(1-2 \lambda_{n} c_{2}\right) \gamma_{n}\left\|t^{n}-y^{n}\right\|^{2} \\
\leq & \alpha_{n}\left\|u-x^{*}\right\|^{2}+\left\|x^{n}-x^{*}\right\|^{2}-\left\|x^{n+1}-x^{*}\right\|^{2} \\
= & \alpha_{n}\left\|u-x^{*}\right\|^{2}+\left(\left\|x^{n}-x^{*}\right\|-\left\|x^{n+1}-x^{*}\right\|\right)\left(\left\|x^{n}-x^{*}\right\|+\left\|x^{n+1}-x^{*}\right\|\right) \\
\leq & \alpha_{n}\left\|u-x^{*}\right\|^{2}+\left(\left\|x^{n}-x^{n+1}\right\|\right)\left(\left\|x^{n}-x^{*}\right\|+\left\|x^{n+1}-x^{*}\right\|\right) .
\end{aligned}
$$

From $\lim _{n \rightarrow \infty} \alpha_{n}=0$ and (3.25), it follows

$$
\lim _{n \rightarrow \infty}\left\|t^{n}-y^{n}\right\|=0 \text {. }
$$

Theorem 3.3. Let $C$ be a nonempty closed convex subset of a real Hilbert space $H$. Suppose that assumptions (i)-(v) hold, for each $x \in C, f(x, \cdot)$ is strongly convex with constant $\delta>0$ on $C$ and $T$ is nonexpansive on $C$. Then the sequences $\left\{x^{n}\right\},\left\{y^{n}\right\}$ and $\left\{t^{n}\right\}$ generated by Algorithm 2.2 converge strongly to the same point $\bar{x}$ provided $\lim _{n \rightarrow \infty}\left\|x^{n}-y^{n}\right\|=0$, where

$$
\bar{x}=\operatorname{Pr}_{\operatorname{Sol}(f, C) \cap F i x(T)}(u) .
$$


Proof. It follows from Lemma 3.1 that

$$
\left\|t^{n}-x^{*}\right\| \leq\left\|x^{n}-x^{*}\right\| \quad \forall n \geq 0,
$$

and hence, we have

$$
\begin{aligned}
\left\|T\left(x^{n}\right)-x^{n}\right\| \leq & \left\|T\left(x^{n}\right)-T\left(t^{n}\right)\right\|+\left\|T\left(t^{n}\right)-x^{n+1}\right\|+\left\|x^{n+1}-x^{n}\right\| \\
\leq & \left\|x^{n}-t^{n}\right\|+\left\|T\left(t^{n}\right)-x^{n+1}\right\|+\left\|x^{n+1}-x^{n}\right\| \\
\leq & \left\|x^{n}-t^{n}\right\|+\alpha_{n}\left\|T\left(t^{n}\right)-u\right\|+\beta_{n}\left\|T\left(t^{n}\right)-x^{n}\right\|+\left\|x^{n+1}-x^{n}\right\| \\
\leq & \left\|x^{n}-t^{n}\right\|+\alpha_{n}\left\|T\left(t^{n}\right)-u\right\|+\beta_{n}\left\|T\left(t^{n}\right)-T\left(x^{n}\right)\right\| \\
& +\beta_{n}\left\|T\left(x^{n}\right)-x^{n}\right\|+\left\|x^{n+1}-x^{n}\right\| \\
\leq & \left\|x^{n}-t^{n}\right\|+\alpha_{n}\left\|T\left(t^{n}\right)-u\right\|+\beta_{n}\left\|t^{n}-x^{n}\right\|+\beta_{n}\left\|T\left(x^{n}\right)-x^{n}\right\| \\
& +\left\|x^{n+1}-x^{n}\right\| .
\end{aligned}
$$

Consequently, from Lemma 3.2 and $\lim _{n \rightarrow \infty} \beta_{n} \in(0,1)$, it follows that

$$
\lim _{n \rightarrow \infty}\left\|T\left(x^{n}\right)-x^{n}\right\|=0 \text {. }
$$

Then, we also have

$$
\begin{aligned}
\left\|T\left(t^{n}\right)-t^{n}\right\| & \leq\left\|T\left(t^{n}\right)-T\left(x^{n}\right)\right\|+\left\|T\left(x^{n}\right)-x^{n}\right\|+\left\|x^{n}-t^{n}\right\| \\
& \leq\left\|t^{n}-x^{n}\right\|+\left\|T\left(x^{n}\right)-x^{n}\right\|+\left\|x^{n}-t^{n}\right\| \\
& \rightarrow 0 \text { as } n \rightarrow \infty .
\end{aligned}
$$

Since $\left\{x^{n}\right\}$ is bounded, there exists a subsequence $\left\{x^{n_{j}}\right\}$ of $\left\{x^{n}\right\}$ so that

$$
\limsup _{n \rightarrow \infty}\left\langle u-x^{*}, x^{n}-x^{*}\right\rangle=\lim _{j \rightarrow \infty}\left\langle u-x^{*}, x^{n_{j}}-x^{*}\right\rangle
$$

where $x^{*}:=\operatorname{Pr}_{\operatorname{Sol}(f, C) \cap F i x(T)}(u)$. Without loss of generality, we may further assume that $\left\{x^{n_{j}}\right\}$ converges weakly to $\bar{x} \in H$. Hence, (3.28) reduces to

$$
\limsup _{n \rightarrow \infty}\left\langle u-x^{*}, x^{n}-x^{*}\right\rangle=\left\langle u-x^{*}, \bar{x}-x^{*}\right\rangle .
$$

From Lemma 2.4, (3.26) and $x^{n_{j}} \rightarrow \bar{x}$ as $j \rightarrow \infty$, it follows

$$
T(\bar{x})=\bar{x} \text {. }
$$

In fact, assume that $\bar{x} \notin F i x(T)$. From Opial's condition in [13], we have

$$
\begin{aligned}
\liminf _{j \rightarrow \infty}\left\|t^{n_{j}}-\bar{x}\right\| & <\liminf _{j \rightarrow \infty}\left\|t^{n_{j}}-T(\bar{x})\right\| \\
& \leq \liminf _{j \rightarrow \infty}\left(\left\|t^{n_{j}}-T\left(t^{n_{j}}\right)\right\|+\left\|T\left(t^{n_{j}}\right)-T(\bar{x})\right\|\right) \\
& =\liminf _{j \rightarrow \infty}\left\|T\left(t^{n_{j}}\right)-T(\bar{x})\right\| \\
& \leq \liminf _{j \rightarrow \infty}\left\|t^{n_{j}}-\bar{x}\right\| .
\end{aligned}
$$

This is a contradiction. Thus, $\bar{x}=T(\bar{x})$.

From Lemma 3.2 and $x^{n_{j}} \rightarrow \bar{x}$ as $j \rightarrow \infty$, it follows

$$
y^{n_{j}} \rightarrow \bar{x}, t^{n_{j}} \rightarrow \bar{x} \text { as } j \rightarrow \infty \text {. }
$$


Then, from (3.10), $\lim _{n \rightarrow \infty} \lambda_{n}=\lambda \in(0,1)$ and assumptions of $f$, it follows

$$
\lambda_{n_{j}}\left(f\left(x^{n_{j}}, y\right)-f\left(x^{n_{j}}, y^{n_{j}}\right)\right) \geq\left\langle y^{n_{j}}-x^{n_{j}}, y^{n_{j}}-y\right\rangle \quad \forall y \in C,
$$

and when $j \rightarrow \infty$, we have $f(\bar{x}, y) \geq 0$ for all $y \in C$. It means that $\bar{x} \in$ $\operatorname{Sol}(f, C)$. Combining this and (3.30), we have

$$
\bar{x} \in \operatorname{Sol}(f, C) \cap \operatorname{Fix}(T) .
$$

Then, it is easy to see that

$$
\left\langle u-x^{*}, \bar{x}-x^{*}\right\rangle \leq 0 .
$$

Thus, combining with (3.29), we have

$$
\limsup _{n \rightarrow \infty}\left\langle u-x^{*}, x^{n}-x^{*}\right\rangle \leq 0
$$

Now, with $x^{*}=\operatorname{Pr}_{\operatorname{Sol}(f, C) \cap F i x(T)}(u)$, from $\left\|t^{n}-x^{*}\right\| \leq\left\|x^{n}-x^{*}\right\|$, Lemma 3.1 and

$$
\langle x, y\rangle \leq \frac{1}{2}\left(\|x\|^{2}+\|y\|^{2}\right) \quad \forall x, y \in H
$$

it implies

$$
\begin{aligned}
& \left\|x^{n+1}-x^{*}\right\|^{2} \\
= & \left\langle\alpha_{n} u+\beta_{n} x^{n}+\gamma_{n} T\left(t^{n}\right)-x^{*}, x^{n+1}-x^{*}\right\rangle \\
= & \alpha_{n}\left\langle u-x^{*}, x^{n+1}-x^{*}\right\rangle+\beta_{n}\left\langle x^{n}-x^{*}, x^{n+1}-x^{*}\right\rangle \\
& +\gamma_{n}\left\langle T\left(t^{n}\right)-x^{*}, x^{n+1}-x^{*}\right\rangle \\
\leq & \alpha_{n}\left\langle u-x^{*}, x^{n+1}-x^{*}\right\rangle+\frac{\beta_{n}}{2}\left(\left\|x^{n}-x^{*}\right\|^{2}+\left\|x^{n+1}-x^{*}\right\|^{2}\right) \\
& +\frac{\gamma_{n}}{2}\left(\left\|T\left(t^{n}\right)-x^{*}\right\|^{2}+\left\|x^{n+1}-x^{*}\right\|^{2}\right) \\
\leq & \alpha_{n}\left\langle u-x^{*}, x^{n+1}-x^{*}\right\rangle+\frac{\beta_{n}}{2}\left(\left\|x^{n}-x^{*}\right\|^{2}+\left\|x^{n+1}-x^{*}\right\|^{2}\right) \\
& +\frac{\gamma_{n}}{2}\left(\left\|t^{n}-x^{*}\right\|^{2}+\left\|x^{n+1}-x^{*}\right\|^{2}\right) \\
\leq & \alpha_{n}\left\langle u-x^{*}, x^{n+1}-x^{*}\right\rangle+\frac{1}{2}\left(1-\alpha_{n}\right)\left(\left\|x^{n}-x^{*}\right\|^{2}+\left\|x^{n+1}-x^{*}\right\|^{2}\right), \\
\leq & \alpha_{n}\left\langle u-x^{*}, x^{n+1}-x^{*}\right\rangle+\left(1-\alpha_{n}\right)\left\|x^{n}-x^{*}\right\|^{2} .
\end{aligned}
$$

This implies that

$$
\left\|x^{n+1}-x^{*}\right\|^{2} \leq\left(1-\alpha_{n}\right)\left\|x^{n}-x^{*}\right\|^{2}+\alpha_{n} \beta_{n},
$$

where $\beta_{n}:=\left\langle x^{n+1}-x^{*}, u-x^{*}\right\rangle$. Then, from an application of Lemma 2.6 and (3.31), it yields that $\lim _{n \rightarrow \infty}\left\|x^{n}-x^{*}\right\|=0$. From Lemma 3.1, it follows $\lim _{n \rightarrow \infty}\left\|y^{n}-x^{*}\right\|=0$ and $\lim _{n \rightarrow \infty}\left\|t^{n}-x^{*}\right\|=0$. 


\section{References}

[1] P. N. Anh, A logarithmic quadratic regularization method for solving pseudomonotone equilibrium problems, Acta Mathematica Vietnamica 34 (2009), 183-200.

[2] _ An LQP regularization method for equilibrium problems on polyhedral, Vietnam Journal of Mathematics 36 (2008), 209-228.

[3] P. N. Anh, L. D. Muu, V. H. Nguyen, and J. J. Strodiot, Using the Banach contraction principle to implement the proximal point method for multivalued monotone variational inequalities, J. Optim. Theory Appl. 124 (2005), no. 2, 285-306.

[4] E. Blum and W. Oettli, From optimization and variational inequalities to equilibrium problems, Math. Student 63 (1994), no. 1-4, 123-145.

[5] P. Daniele, F. Giannessi, and A. Maugeri, Equilibrium Problems and Variational Models, Kluwer, 2003

[6] J. K. Kim, S. Y. Cho, and X. Qin, Hybrid projection algorithms for generalized equilibrium problems and strictly pseudocontractive mappings, J. Inequal. Appl. 2010 (2010), Art. ID 312602, 18 pp.

[7] _ Some results on generalized equilibrium problems involving strictly pseudocontractive mappings, Acta Math. Scientia 31(5) (2011), 2041-2057.

[8] J. K. Kim and N. Buong, Regularization inertial proximal point algorithm for monotone hemicontinuous mapping and inverse strongly monotone mappings in Hilbert spaces, J. Inequal. Appl. 2010 (2010), Art. ID 451916, 10 pp.

[9] G. M. Korpelevich, An extragradient method for finding saddle points and for other problems, Ekonom. i Mat. Metody 12 (1976), no. 4, 747-756.

[10] P. Kumama, N. Petrot, and R. Wangkeeree, A hybrid iterative scheme for equilibrium problems and fixed point problems of asymptotically $k$-strict pseudo-contractions, J. Comput. Appl. Math. 233 (2010), no. 8, 2013-2026.

[11] G. Li and J. K. Kim, Demiclosedness principle and asymptotic behavior for nonexpansive mappings in metric spaces, Appl. Math. Lett. 14 (2001), no. 5, 645-649.

[12] N. Nadezhkina and W. Takahashi, Weak convergence theorem by an extragradient method for nonexpansive mappings and monotone mappings, J. Optim. Theory Appl. 128 (2006), no. 1, 191-201.

[13] Z. Opial, Weak convergence of the sequence of successive approximations for nonexpansive mapping, Bull. Amer. Math. Soc. 73 (1967), 591-597.

[14] J. W. Peng, Iterative algorithms for mixed equilibrium problems, strict pseudocontractions and monotone mappings, J. Optim. Theory Appl. 144 (2010), no. 1, 107-119.

[15] Y. Shehu, Fixed point solutions of generalized equilibrium problems for nonexpansive mappings, J. Comput. Appl. Math. 234 (2010), no. 3, 892-898.

[16] S. Takahashi and W. Takahashi, Viscosity approximation methods for equilibrium problems and fixed point problems in Hilbert spaces, J. Math. Anal. Appl. 331 (2007), no. $1,506-515$.

[17] W. Takahashi and M. Toyoda, Weak convergence theorems for nonexpansive mappings and monotone mappings, J. Optim. Theory Appl. 118 (2003), no. 2, 417-428.

[18] S. Wang and B. Guo, New iterative scheme with nonexpansive mappings for equilibrium problems and variational inequality problems in Hilbert spaces, J. Comput. Appl. Math. 233 (2010), no. 10, 2620-2630.

[19] H. K. Xu, Viscosity method for hierarchical fixed point approach to variational inequalities, Taiwanese J. Math. 14 (2010), no. 2, 463-478.

[20] H. K. Xu and T. H. Kim, Convergence of hybrid steepest-descent methods for variational inequalities, J. Optim. Theory Appl. 119 (2003), no. 1, 185-201.

[21] Y. Yao, Y. C. Liou, and Y. J. Wu, An extragradient method for mixed equilibrium problems and fixed point problems, Fixed Point Theory Appl. 2009 (2009), Art. ID 632819, 15 pp. 
[22] L. C. Zeng and J. C. Yao, Strong convergence theorem by an extragradient method for fixed point problems and variational inequality problems, Taiwanese J. Math. 10 (2006), no. $5,1293-1303$.

Jong KYU KIM

Department of Mathematics

KyUNGNAM University

MASAN 631-701, KOREA

E-mail address: jongkyuk@kyungnam.ac.kr

Pham Ngoc Anh

Posts and Telecommunications Institute of Technology

HanoI, Vietnam

E-mail address: anhpn@ptit.edu.vn

YOUNG MAN NAM

Department of Mathematics

KYUNGNAM UNIVERSITY

MASAN 631-701, Korea

E-mail address: nym4953@kyungnam.ac.kr 